\title{
Determinants of response and resistance to cytokine-induced killer cell immunotherapy for advanced solid tumors
}

\author{
Wenjun Wang ${ }^{1}$, Chengzhi Zhou ${ }^{1}$, Sipei $\mathrm{Wu}^{2}$, Yalei Zhang ${ }^{1}$, Puyi Lie ${ }^{1}$, Shunjun Jiang ${ }^{1}$, \\ Yiping Jiang ${ }^{1}$, and Jianxing $\mathrm{He}^{1}$ \\ ${ }^{1}$ First Affiliated Hospital of Guangzhou Medical College \\ ${ }^{2}$ Guangdong Provincial People's Hospital
}

September 10, 2020

\begin{abstract}
This study aimed to understand the influence of naïve T cells in cytokine-induced killer (CIK) cell immunotherapy. A total of 68 patients with advanced solid tumors received activated autologous CIK cells weekly. Objective responses (complete or partial responses, $\mathrm{CR} / \mathrm{PR})$ were observed in 11 patients $(16.2 \%, 11 / 68)$, with five of 11 responses lasting more than 8 months, and in 16 patients with SD $(23.5 \%, 16 / 68)$. The absolute number of CD4+ naïve T cells in patients who achieved CR/PR was higher in $\mathrm{SD}$ and $\mathrm{PD}$ patients (CR/PR vs $\mathrm{SD}$ vs $\mathrm{PD}=90$ vs 149 vs 226 cells/ $\mu \mathrm{L})$, as were the absolute number of CD8+ naïve T cells $(\mathrm{CR} / \mathrm{PR}$ vs $\mathrm{SD}$ vs $\mathrm{PD}=47$ vs 60 vs 103 cells/ $\mu \mathrm{L}$ ). Patients with high absolute numbers of naïve T cells (\{greater than or equal to 298 cells $/ \mu \mathrm{L}$ in naïve CD4+ and \{greater than or equal to 156 cells/ $\mu \mathrm{L}$ in naïve CD8+ cells) had a better response to ACT (PFS=8 months vs 5 months). In this study, we found that Patients with a high absolute number of naïve $\mathrm{T}$ cells in circulating blood had a better response to ACT, which showed the potential of naïve $\mathrm{T}$ cells as a biomarker for the response to ACT.
\end{abstract}

Determinants of response and resistance to cytokine-induced killer cell immunotherapy for advanced solid tumors

Wenjun Wang ${ }^{1 *}$, Chengzhi Zhou ${ }^{1}$, Sipei Wu ${ }^{2}$, Yalei Zhang ${ }^{1}$, Puyi Lie $^{1}$,Shunjun Jiang $^{1}$, Yiping Jiang ${ }^{1}$, Jianxing $\mathrm{He}^{1^{*}}$

1. State Key Laboratory of Respiratory Diseases, Guangzhou Institute of Respiratory Disease, The First Affiliated Hospital of Guangzhou Medical University, Guangzhou 510120, China

2. Guangdong Lung Cancer Institute, Guangdong General Hospital and Guangdong Academy of Medical Sciences, Guangzhou 510080, China

Correspondence to: Wenjun Wang and Jianxing He, State Key Laboratory of Respiratory Diseases, Guangzhou Institute of Respiratory Disease, the First Affiliated Hospital of Guangzhou Medical University, Guangzhou, 510120, China, E-mail: wwj01@foxmail.com,hejx@vip.163.com.

This study aimed to understand the influence of naïve T cells in cytokine-induced killer (CIK) cell immunotherapy. A total of 68 patients with advanced solid tumors received activated autologous CIK cells weekly. Objective responses (complete or partial responses, CR/PR) were observed in 11 patients $(16.2 \%, 11 / 68)$, with five of 11 responses lasting more than 8 months, and in 16 patients with SD $(23.5 \%, 16 / 68)$. The absolute number of CD4+ naïve T cells in patients who achieved CR/PR was higher in SD and PD patients (CR/PR vs $\mathrm{SD}$ vs $\mathrm{PD}=90$ vs 149 vs 226 cells/ $\mu \mathrm{L})$, as were the absolute number of $\mathrm{CD} 8+$ naïve $\mathrm{T}$ cells $(\mathrm{CR} / \mathrm{PR}$ vs $\mathrm{SD}$ vs $\mathrm{PD}=47$ vs 60 vs 103 cells $/ \mu \mathrm{L}$ ). Patients with high absolute numbers of naïve $\mathrm{T}$ cells (\{greater than or equal to 298 cells $/ \mu \mathrm{L}$ in naïve CD4+ and greater than or equal to 156 cells/ $\mu \mathrm{L}$ in naïve CD $8+$ cells) had a better response to $\mathrm{ACT}$ ( $\mathrm{PFS}=8$ months vs 5 months). In this study, we found that Patients with a 
high absolute number of naïve T cells in circulating blood had a better response to ACT, which showed the potential of naïve $\mathrm{T}$ cells as a biomarker for the response to ACT.

Keywords: Adoptive $\mathrm{T}$ cell therapy $(\mathrm{ACT})$, cytokine-induced killer cell immunotherapy, naïve $\mathrm{T}$ cells,immunological monitoring

\section{Introduction}

Adoptive T cell therapy (ACT) is a major immunotherapy and has been shown to be a powerful approach to cancer treatment [1]. Cytokine-induced killer (CIK) cells are polyclonal T effector cells sharing immunological properties and receptors with natural killer (NK) cells. They are attracting increasing interest for their ability to perform non-MHC-restricted cytolytic activities towards susceptible autologous and allogeneic cancer cells [2-3]. CIK cell treatment has an adjuvant immunomodulatory effect by prolonging survival in several types of cancer patients who undergo curative treatment [4]. Adjuvant immunotherapy with autologous cytokineinduced killer cells have evidence clinical effects in hepatocellular carcinoma [5]. Moreover, a phase I study result showed that the activity of PD-1 blockade-activated CIK cells in patients with advanced solid tumors was still unsatisfactory, as the objective response rate (ORR) across the 31 enrolled patients was $22.5 \%$, with only two patients (6.4\%) showing a complete response (CR) and five patients (16.1\%) showing a partial response (PR) [6]. Therefore, a detailed analysis is necessary to determine which patients will have a good response to CIK cell therapy that maintains sustained antitumor effects.

The state of $\mathrm{T}$ cell differentiation is an important factor that influences immune activity after ACT. It is likely that innate humoral and cellular immune deficiencies, including inherent $\mathrm{T}$ cell defects, lead to $\mathrm{T}$ cell exhaustion and treatment failure. Cultured T cells with the phenotype of CD45RO-, CCR7+, CD45RA+ and CD62L + are characteristic of naive T cells. Naive T cells are a subset of less differentiated $\mathrm{T}$ cells with strong self-renewal and multipotent capacity to derive effector $\mathrm{T}$ cells that are specific for multiple viral and self-tumor antigens [7-9]. Naive T cells resist terminal differentiation and maintain high replicative potential that have shown great efficacy in clinical trials, so they may be a superior subset for use in adoptive immunotherapy. For ACT, because most protocols require that peripheral blood lymphocytes be cultured for at least 12-14 days ex vivo, we hypothesized that the number of naive T cells among patients' peripheral blood lymphocytes may influence the culture results including $\mathrm{T}$ cell cytotoxicity and amplification. ACT utilizes CIK cells, genetically engineered $\mathrm{T}$ cells with chimeric antigen receptors (CARs), $\mathrm{T}$ cells and $\mathrm{T}$ cell receptor (TCR) therapy, but the blood-based biomarkers that may be useful for predicting clinical response are still completely unclear. CD27+PD-1-CD8+ T cell populations of less differentiated cells have been used as blood-based biomarkers to predict clinical response in CAR T cell therapy [10]. Pre-clinical studies have also reported that naive and less differentiated $\mathrm{T}$ cells have strong cell activity for long-term immune response [11-16]. Moreover, the increased persistence of adoptively transferred cells appears to be dependent upon the acquisition of naive or less differentiated populations [17-20].

Naive and less differentiated $\mathrm{T}$ cells have strong self-renewal and multipotent capacity to derive effector $\mathrm{T}$ cells and are specific for multiple viral and self-tumor antigens [21]. Blood-based biomarkers are important for predicting clinical response to immunotherapy. In CIK cell therapy, it is unclear whether naive and less differentiated $\mathrm{T}$ cells will work as blood-based biomarkers to determine which patients will have a good response. We hypothesized that patients with high absolute numbers of naive $\mathrm{T}$ cells in circulating blood will have a better response to CIK cell therapy. This clinical trial was therefore designed to understand whether naive $\mathrm{T}$ cells in circulating blood can serve as pretreatment biomarkers to determine which patients will have a better response to CIK cell therapy.

\section{Materials and Methods}

\section{Cells and cell cultures}

The generation of CIK cells was based on a previous study [22]. Blood $(80 \mathrm{~mL})$ was obtained from heparinized peripheral blood of all the patients. Peripheral blood mononuclear cells (PBMCs) were isolated by FicollHypaque gradient centrifugation. Cells were cultured in X-15 medium containing $2 \%$ autologous serum and 
adhered for 1 hour. The suspension cells were stimulated with $1000 \mathrm{U} / \mathrm{ml}$ IFN-gamma for the first 24 hours, then induced into CIK cells with $100 \mathrm{ng} / \mathrm{ml}$ OKT-3, $1000 \mathrm{U} / \mathrm{ml}$ RHIL-2 and $100 \mathrm{U} / \mathrm{ml} \mathrm{IL-1} \alpha$. At 14 days, the fraction of CIK cells were collected to assess their number, phenotype, and viability of cells, and to test for possible contamination by bacteria, fungi, or endotoxins. Vitalities of CIKs determined by trypan blue staining, CIKs activity and CD3+CD45+ T cells were accounted for more than $95 \%$ and $90 \%$ of total cells. Then, autologous CIK (1.0-1.5*10 ${ }^{10}$ cells) were transferred to patients via intravenous infusion.

\section{Flow cytometry}

Whole blood $(100 \mu \mathrm{L})$ or $1 \times 10^{6}$ CIK infusion cells were stained for cell surface markers to analyze $\mathrm{T}$ cell differentiation status. The following antibodies were used: anti-CD45RA-FITC; anti-CCR7-PE, antiCD3-PC7; anti-CD8-APC, anti-CD45RO-PC7 (Tube1); anti-CD45-APC, anti-CD25-PE, anti-Helios-FITC; anti-CD4-PC7; anti-FoxP3-PC5.5 (Tube2); anti-CD45-APC, anti-CD8-PE, anti-CD56-FITC; anti-CD4-PC7; anti-CD3-PC5.5 (Tube3); anti-CD45-APC, anti-CD8-PE, anti-HLA-DR-FITC; anti-CD4-PC7; anti-CD3PC5.5 (Tube4); anti-CD45-APC, anti-CD16-PE, anti-CD1c-FITC; anti-CD11c-PC7. All antibodies were titrated before use, and fluorescence-minus-one (FMO) controls were created for each antibody panel to set gates for positive events. For anti-lineage-PC5.5 (Tube5), cells were washed with phosphate-buffered saline (PBS) and stained for viability using LIVE/DEAD Fixable Violet (Molecular Probes) for 15 min, washed once and resuspended in fluorescence-activated cell sorting (FACS) buffer consisting of PBS, 1\% BSA and $5 \mathrm{mM}$ EDTA. Cells were then incubated with the above indicated antibodies for $1 \mathrm{~h} \mathrm{at} 4^{\circ} \mathrm{C}$. Samples were washed three times with FACS buffer and fixed in 1\% paraformaldehyde. Positively-stained cells were differentiated from background using FMO controls. Flow cytometry was performed using the BD LSR Fortessa flow cytometer. Analysis was performed using Flowjo software (Tree Star Inc. version 10.1). Absolute cell numbers were determined by relating the $\mathrm{CD} 45^{+}$cell count from flow cytometry to the total leucocyte count.

\section{Patients and treatment plans}

A total of 68 patients were selected with advanced solid tumors (including 42 lung cancers, 14 liver cancers, 9 colon cancers and 3 renal carcinomas) who had experienced disease progression after at least one previous course of tumor-appropriate treatment for advanced or metastatic disease. Other inclusion criteria included stopping any cancer therapy before enrollment, an age of 18 to 75 years, a life expectancy greater than 12 weeks, an Eastern Cooperative Oncology Group (ECOG) performance status of 0 or 1, adequate organ function, and lesions that could be evaluated using the Response Evaluation Criteria in Solid Tumors (RECIST) guidelines. The following exclusion criteria were applied: previous treatment with anti-CTLA-4 or anti-PD-1/PD-L1 therapy, any form of primary immunodeficiency or history of autoimmune disease, ongoing systemic infections and concurrent systemic steroid therapy, and recruitment into other clinical trials. All participating patients provided informed consent.

This open-label, phase I \& II trial was approved by the Review Board and Clinical Research Ethics Committee of The First Affiliated Hospital of Guangzhou Medical University. It's registered atwww.chictr.org.cn.(ChiCTR-OIC-17011679). All methods and procedures employed by this study were performed in accordance with Good Clinical Practice guidelines and were in accordance with the principles of the Declaration of Helsinki and local laws. All authors had access to the study data and reviewed and approved the final manuscript. The enrolled patients received intravenous infusions of autologous CIK cells. All participants received at least eight cycles of infusions (four cycles at weekly intervals followed by four cycles every 2 weeks) or received cycles until they experienced disease progression or unacceptable adverse events (AEs) or withdrew consent. Patients with disease progression were removed from the study and then received multidisciplinary synthetic therapy or were recruited into other clinical trials according to the recommendations of physicians. If the patients had an objective response or stable disease after treatment, they were viewed as eligible to receive additional cycles of maintenance treatment every 2 weeks. The design and procedures used in the clinical trial are shown in Figure 1.

\section{Treatment-Related Toxicities}


The most common AEs arising from treatment with CIK cells during the study were fever; all fevers rose no higher than $38^{\circ} \mathrm{C}$ and spontaneously resolved within $12 \mathrm{~h}$. Additional AEs observed were decreased albumin levels, increased serum AST and ALT levels, anemia, leukopenia, thrombocytopenia, chills, hypothyroidism and vitiligo. All grades of treatment-related AEs occurred in 25 of 68 patients (36.8\%), and most of the cytotoxicity assay results were grade 1 or 2 (22 of 25 patients, $88 \%$ ). Grade 3 or 4 treatment-related AEs were observed in only 3 patients (12\%). One NSCLC and one CRC patient were observed with grade 3 fever, and grade 3 chill was observed in another HCC patient during treatment (Table 1).

\section{$\mathbf{T}$ cell proliferation assay}

The PBMCs were obtained from heparinized peripheral blood of patients. Cells were put into 96-well plates at $1 \times 10^{4}$ cells/well and cultured in X-VIVO 15 containing $2 \%$ autologous serum and allowed to adhere for 1 $\mathrm{h}$. The suspended cells were then collected and induced to become CIK cells using $1,000 \mathrm{U} / \mathrm{mL}$ rhIFN- $\gamma$ for the first $24 \mathrm{~h}$ followed by stimulation with $100 \mathrm{ng} / \mathrm{mL}$ OKT-3, $1000 \mathrm{U} / \mathrm{ml}$ rhIL-2 and $100 \mathrm{U} / \mathrm{mL}$ IL- $1 \alpha$. The cells were counted $1,3,5,7,9,12$ and 15 days later. All experiments were performed at least three times and the mean was calculated.

\section{Statistical analysis}

The graphs represent the mean value+standard deviation (SD), unless otherwise indicated. A student's $t$ test for paired data, Wilcoxon rank-sum test, or a one-way ANOVA were performed using GraphPad Prism version 5.0 (GraphPad Software). Descriptive statistics were used to summarize the patient characteristics, treatment-related AEs, overall responses and CIK cell phenotypes. The Mann-Whitney U-test was used to compare continuous variables, and Fisher's exact test was used for categorical variables between groups. Survival rates were calculated using the Kaplan-Meier method. Overall survival (OS) was defined from the date of first treatment to the date of death resulting from any cause or the date of last follow-up. Progressionfree survival (PFS) was calculated from the time of first treatment to the time of first disease progression or last follow-up. A difference of $\mathrm{p}<0.05$ was considered statistically significant in all the analyses.

\section{Results}

\section{Patients with a high frequency of naïve $\mathrm{T}$ cells in circulating blood have better responses to CIK immunotherapy.}

To identify the specific factors in heparinized peripheral blood that predict why only certain patients have strong responses to CIK treatment, we studied 68 patients with advanced solid tumors (including 42 lung cancers, 14 liver cancers, nine colon cancers and three renal carcinomas) who received activated autologous CIK cell treatment (Figure 1). The patient clinical characteristics are shown in Table 1. As shown in our data, efficacy of treatment was not related to patient age, prior therapy, peripheral tumor burden, tumor histology, ECOG PS or other typical factors (Table 1). We first tested the frequency of 11 leucocyte subsets in the heparinized peripheral blood of patients before CIK treatment. The absolute and proportional medians of the 11 leucocyte subsets in different clinical responses are presented in Table 2. As shown in the hierarchical clustering of partial subsets of proportional data across all samples analyzed in a heatmap representation, we divided the patient into three clusters of $\mathrm{PD}, \mathrm{SD}$ and $\mathrm{CR} / \mathrm{PR}$. Naïve $\mathrm{CD} 4^{+}$and $\mathrm{CD} 8^{+}$ $\mathrm{T}$ cells subsets contributed to the separation with higher frequencies (marked in green) observed in samples from CR/PR patients, whereas samples from PD individuals contained fewer proportions (Figure 2A). The naïve $\mathrm{CD} 4^{+}$and $\mathrm{CD} 8^{+} \mathrm{T}$ cell subsets were identified by surface markers: $\mathrm{CD} 4^{+} \mathrm{CD} 45 \mathrm{RA}^{+} \mathrm{CCR}^{+} \mathrm{CD}^{+} 2 \mathrm{~L}^{+}$

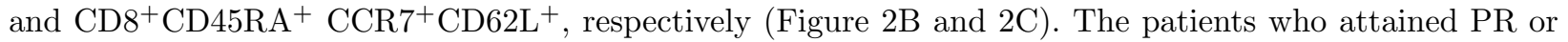
CR by CIK therapy had higher absolute numbers of naïve $\mathrm{CD} 4^{+}$and $\mathrm{CD} 8^{+} \mathrm{T}$ cells in heparinized peripheral blood (naïve $\mathrm{CD}^{+}$means: $\mathrm{PD}$ vs $\mathrm{SD}$ vs $\mathrm{CR} / \mathrm{PR}=73$ vs 108 vs 202 ; naïve $\mathrm{CD} 8^{+}$means: $\mathrm{PD}$ vs $\mathrm{SD}$ vs $\mathrm{CR} / \mathrm{PR}=31$ vs 62 vs 90 ) (Figure $2 \mathrm{D}$ and $2 \mathrm{~F}$ ). The frequency and absolute number of naïve $\mathrm{CD} 4^{+} \mathrm{T}$ cells were significantly higher in the subset of patients who reached CR/PR than in PD or SD patients after CIK therapy (Figure $2 \mathrm{E}$ and $2 \mathrm{~F}$ ). Moreover, the absolute number of naïve $\mathrm{CD} 8^{+}$cells was also higher in the subset of patients who reached CR/PR than in PD or SD patients, but the frequency were no statistical differences between $\mathrm{CR} / \mathrm{PR}$ patients and $\mathrm{PD} / \mathrm{SD}$ patients(Figure $2 \mathrm{E}$ and $1 \mathrm{~F}$ ). To summarize, our results 
indicated that patients who reached CR/PR after CIK therapy had higher absolute numbers of naïve CD4 ${ }^{+}$ and naïve $\mathrm{CD} 8^{+} \mathrm{T}$ cells.

The intrinsic potency of naïve $T$ cells from heparinized peripheral blood drives the response to CIK cell therapy.

$\mathrm{T}$ cells obtained from healthy donors and patients show variable proportions of T-cell subsets. Both CD4 ${ }^{+}$ and $\mathrm{CD}^{+}$naïve cells $(\mathrm{Tn})$ comprised a proportionately higher absolute number of healthy donor compared with patients $\left(298\right.$ cells $/ \mu \mathrm{L}$ vs. 156 cells $/ \mu \mathrm{L}, \mathrm{p}<0.001$, for $\mathrm{CD} 4^{+} \mathrm{Tn}$, and 157 cells $/ \mu \mathrm{L}$ vs. 56 cells $/ \mu \mathrm{L}, \mathrm{p}$ $<0.001$, for $\left.\mathrm{CD} 8^{+} \mathrm{Tn}\right)$. When there was only higher percentage of healthy donor compared with patients in $\mathrm{CD} 8^{+}$naïve cells $(44.6 \%$ vs. $17.5 \%, \mathrm{p}<0.001)$ (Figure $3 \mathrm{~A}$ and $3 \mathrm{~B}$ ). We next compared the proliferation of CIK cells from healthy donors and patients of absolute number of naïve CD4 ${ }^{+}$and naïve CD ${ }^{+}$cells in peripheral blood. We found that $\mathrm{T}$ cells from healthy donors exhibited a significantly greater proportion of naïve $\mathrm{T}$ cells compared to patients (Figure $3 \mathrm{C}$ and 3D). To understand whether CIK cells amplified from healthy donors with high naïve $\mathrm{T}$ cell counts had more activity and cytotoxicity. The proliferation of CIK cells that was associated with progressive differentiation during 15 days culture in vitro, and the number of $\mathrm{T}$ cells displaying an immune exhaustion phenotype such as CD $3^{+} \mathrm{PD}-1^{+} \mathrm{Tim}-3^{+}$were significantly increased in samples from patients, but not in samples from healthy donors (Figure 3E ). However the percentage of proliferating CIK cells $\left(\mathrm{CD} 3^{+} \mathrm{CD} 56^{+} \mathrm{Ki} 67^{+}\right)$from healthy donors was significantly greater than patients (Figure 3F). Moreover, cytotoxicity assays found that the in vitro cytolytic activity of CIK cells after amplification was higher in the samples from healthy donors than in samples from patients (Figure $3 \mathrm{G}$ ). In our study, the frequency of naïve $\mathrm{T}$ cell in the patients with $\mathrm{CR} / \mathrm{PR}$ were higher then in PD or SD. To determine if the higher proliferation rate and cytotoxicity of CIK cells amplified from patients with CR/PR could be maintained as long-term persistent immune activity, we checked the frequency and absolute numbers of naïve $\mathrm{CD} 4^{+}$and $\mathrm{CD} 8^{+}$cells in peripheral blood after 1 and 2 weeks transfer immunotherapy. Our results showed that PR/CR patients maintained the high level of CIK cells $\left(\mathrm{CD} 3{ }^{+} \mathrm{CD} 56^{+}\right)$(Figure $3 \mathrm{H}$ and $\left.2 \mathrm{I}\right)$ and IFN- $\gamma$-producing CIK cells $\left(\mathrm{CD}^{+}{ }^{+} \mathrm{CD} 56^{+} \mathrm{IFN}-\gamma^{+}\right)$(Figure 3J and $\left.3 \mathrm{~K}\right)$ in peripheral blood. In summary, these findings indicated that patients with high absolute number of naïve $\mathrm{CD} 4^{+}$and naïve $\mathrm{CD} 8^{+}$cells could produce more CIK cells which likely play an important role in CIK immunotherapy.

High absolute number of naïve $T$ cells exhibit enhanced potency and persistence in patients.

The CR/PR patients showed a high absolute number of naïve $\mathrm{CD} 4^{+}$and naïve $\mathrm{CD} 8^{+}$cells in peripheral blood. To confirm that naïve $\mathrm{T}$ cells played an important role in the response to CIK immunotherapy, we checked the frequencies and absolute number of $\mathrm{T}$ cell phenotypic markers in CIK immunotherapy patients at days $5,10,15,20,30$ and 35 . We found that patients with a high naïve T cell count ([?]298 cells/ $\mu \mathrm{L}$ in $\mathrm{CD}^{+}$ Tn and [?] 157 cells $/ \mu \mathrm{L}$ in CD $\left.8^{+} \mathrm{Tn}\right)$ maintained a higher frequency of CIK cells(CD3 $\left.{ }^{+} \mathrm{CD} 56^{+}\right)$in peripheral blood after CIK cell immunotherapy (Figure 4A ). The frequency of effector-memory phenotype CIK cells $\left(\mathrm{CD}^{+} \mathrm{CD} 56^{+} \mathrm{IFN}-\gamma^{+}\right)$was higher in patients with high vs low naïve $\mathrm{T}$ cell counts (Figure 4B). However, CIK cells derived from patients with high numbers of naïve $\mathrm{T}$ cells exhibited a lower frequency of the immune exhaustion phenotype $\left(\mathrm{CD} 3^{+} \mathrm{CD} 56^{+} \mathrm{PD}-1^{+} \mathrm{Tim}-3^{+}\right)$compared with patients with low numbers of naïve $\mathrm{T}$ cells on days 5, 15 and 20 after CIK cell immunotherapy (Figure 4D). Moreover, the higher frequency of effector-memory phenotype CIK cells in high count naïve $\mathrm{T}$ cell patients included higher numbers of $\mathrm{CD}^{+} \mathrm{CD} 56^{+} \mathrm{Ki} 67^{+}$cells on treatment days 5, 15 and 20. In summary, these findings indicated that the CIK cells harvested from patients with high absolute numbers of naïve $\mathrm{CD} 4^{+}$and naïve $\mathrm{CD} 8^{+}$cells could produce more CIK cells and maintain sustained antitumor activity.

The absolute number of naïve $\mathrm{T}$ cell populations in patients predicts response to CIK cell therapy.

Based upon the above results that patients with a high absolute number of naïve $\mathrm{CD}^{+}$and naïve $\mathrm{CD} 8^{+}$ cells could produce more CIK cells and maintain sustained antitumor activity, we compared the absolute number of naïve $\mathrm{T}$ cells to healthy donors (Mean $=298$ in naïve $\mathrm{CD}^{+}$and 156 in naïve $\mathrm{CD} 8^{+}$cells) by dividing the patients into groups with high naïve T cell counts (15 of 68) and low naïve T cell counts (53 of 
68). In the overall population, the ORR was $13.2 \%$ (9 of 68 ), while the ORR in patients with high naïve $\mathrm{T}$ cell counts was $40 \%$ ( 6 of 15) compared to $5.7 \%$ (3 of 53) in patients with low naïve T cell counts. Moreover, $4.4 \%$ of patients with high numbers of naïve T cells (3 of 68 ) showed CR and $11.8 \%$ (8 of 68 ) showed PR, but only one patient showed CR among the low naïve T cell-count patients. In addition, $23.5 \%$ (16 of 68 ) of patients achieved SD and a disease control rate (DCR) of 42.6\% (29 of 68). Two NSCLC patients with high numbers of naïve $\mathrm{T}$ cells had greater than $50 \%$ target lesion reduction by 18 weeks (Figure $5 \mathrm{C}$ ), and a patient with high naïve $\mathrm{T}$ cell counts who experienced multiple brain metastases achieved $\mathrm{CR}$ after chemotherapy and treatment with radio frequency ablation (Figure 5C). Among responders, 26.5\% (18 of 68 patients) had responded by month 4, including $21.6 \%$ (11 of 51 ) with low naïve T cell counts and $41.2 \%$ (7 of 17) with high counts. Among responders, $22 \%$ of responses (4 of 18 patients) were ongoing, with response durations ranging from 4 to $14.5+$ months. Among patients with high naïve T cell counts, $42.9 \%$ (3 of 7 ) displayed an ongoing response, including a liver cancer and NSCLC patient with PR who achieved an antitumor response for more than 10 months after eight cycles of autologous CIK cell treatment. In contrast, only one patient with a low naïve $\mathrm{T}$ cell count achieved an ongoing response after eight cycles of autologous CIK cell treatment (Figure 5D). The median PFS was significantly longer in 51 patients with low naïve T cell counts (5 months; $95 \%$ CI 1-9 months) than in 17 patients with high numbers of naive T cells (8 months; $95 \%$ CI $2-14.5$ months; $\mathrm{P}<0.0026$; Figure 5E).

\section{Discussion}

ACT is an important component of anti-tumor therapies. Although CIK cell immunotherapy has been confirmed to be effective in solid tumors, many patients still display no response to ACT. The mechanisms controlling which patients will respond to CIK cell immunotherapy are still unclear. Naive T cells that develop in the thymus have long-term persistence over the human lifespan that could drive immune responses to new antigens. Expansion of immune cells is an essential part of ACT immunotherapies. It is likely that innate humoral and cellular immune deficiencies, including inherent $\mathrm{T}$ cell defects, will lead to $\mathrm{T}$ cell exhaustion and treatment failure. We showed that the patients who reached CR/PR after CIK therapy had higher absolute numbers of naive $\mathrm{CD} 4^{+}$and naive $\mathrm{CD} 8^{+}$cells. Patients who achieved PR or CR by CIK therapy also had higher absolute numbers of naive $\mathrm{CD} 4^{+}$and $\mathrm{CD} 8^{+} \mathrm{T}$ cells in heparinized peripheral blood.

Data from several preclinical immunotherapy studies suggest that the ability of $\mathrm{T}$ cells to engraft following adoptive transfer is related to their state of differentiation, and effector CD8+ $\mathrm{T}$ cells derived from naive rather than memory subsets possess superior traits for ACT [8-11]. Naive T cells present in the periphery since birth are highly proliferative, with long telomeres and high levels of telomerase activity [22-26]. Based on these studies, we investigated whether CIK cells amplified from patients with high naive T cell numbers had more activity and cytotoxicity and found that the proliferation rate of CIK cells from patients with high absolute numbers of naive $\mathrm{T}$ cells was significantly greater than in patients with low numbers of naive $\mathrm{T}$ cells. Our findings showed that patients with high absolute numbers of naive $\mathrm{T}$ cells could produce more CIK cells, indicating that naive T cells play an important role in CIK immunotherapy.

A study of CAR T immunotherapy found that CAR T cells derived from a shorter duration of ex vivo culture could product less differentiated T cells, significantly enhanced effector function and enhanced immunological activity in vivo [27]. To understand whether naive $\mathrm{T}$ cells were a critical factor that could affect the response to CIK immunotherapy, we checked the frequencies and absolute number of $\mathrm{T}$ cell phenotypic markers in CIK immunotherapy patients at days 5, 15 and 20. The frequency of effector-memory phenotype $\mathrm{CD}^{+} \mathrm{T}$ cells $\left(\mathrm{IFN}-\gamma^{+} \mathrm{CD}^{+}\right)$was higher in patients with high vs low numbers of naïve $\mathrm{T}$ cells. The higher frequency of effector-memory phenotype $\mathrm{CD} 8^{+} \mathrm{T}$ cells $\left(\mathrm{IFN}-\gamma^{+} \mathrm{CD} 3^{+}\right)$was associated with higher numbers of $\mathrm{Ki} 67^{+} \mathrm{CD} 3^{+} \mathrm{CD} 56^{+}$cells in these patients. These findings indicated that CIK cells harvested from patients with high absolute numbers of naïve $\mathrm{CD} 4^{+}$and naïve $\mathrm{CD} 8^{+}$cells could produce more CIK cells and maintain sustained antitumor activity.

ACT is a major immunotherapy, as some clinical trials have demonstrated [28, 29]. CIK immunotherapy has potential benefits for several solid tumors including NSCLC; however, the factors that determine which patients will have a positive response to CIK immunotherapy are still unclear. In our study, in order to 
confirm that naïve $\mathrm{T}$ cells play an important role in CIK immunotherapy, we first analyzed the relationship between clinical response and naïve T cell content after CIK treatment. We found that the ORR was $13.2 \%$ (9 of 68), but the ORR in patients with high counts of naïve T cells patients was $35 \%$ (6 of 17) compared to $5.9 \%$ ( 3 of 51) in patients with low counts. Moreover, $2.9 \%$ of patients ( 2 of 68 ) showed CR and $10.3 \%$ ( 7 of 68) showed PR, but only one of the CR patients had a low naïve T cell count. In addition, $23.5 \%$ (16 of 68) of patients achieved SD and a DCR of $42.6 \%$ (29 of 68 ).

In summary, our results suggested that amplification of CIK cells from patients with high numbers of naïve $\mathrm{T}$ cells significantly enhanced effector function, enhanced proliferative capacity and improved anti-tumor efficacy in vivo. The patients with high absolute numbers of naïve $\mathrm{T}$ cells in circulating blood had a better response to ACT, which demonstrated the potential of using pretreatment biomarkers of response in ACT. The frequency of naïve $\mathrm{T}$ cells in circulating blood may serve as a biomarker to identify which patients will have a better response to CIK cell immunotherapy. Our study thus provides critical information to establish highly efficient CIK cell immunotherapy that may be applicable in various clinical settings.

Disclosure of potential conflicts of interest: All authors have declared that they have no conflicts of interest regarding this work.

Acknowledgments: This work was supported by a grant from the National Natural Science Foundation for Young Scholar of China (81402560), the National Natural Science Foundation of China (81572865 and 81472387), and the Guangdong Province Science and Technology Plan Project (2013B021800063).

References

[1] Rosenberg S A, Restifo N P . Adoptive cell transfer as personalized immunotherapy for human cancer. Science.2015;348:62-68.

[2] June C H , Riddell S R , Schumacher T N . Adoptive cellular therapy: A race to the finish line. Science translational medicine.2015;7:280ps7.

[3] De Witte MA, Kierkels GJ, Straetemans T, et al. Orchestrating an immune response against cancer with engineered immune cells expressing $\alpha$ TCRs, CARs, and innate immune receptors: an immunological and regulatory challenge. Cancer Immunol Immunother.2015;64:893-902.

[4] Li JJ, Gu MF, Pan K, et al. Autologous Cytokine-induced Killer Cell Transfusion in Combination With Gemcitabine Plus Cisplatin Regimen Chemotherapy for Metastatic Nasopharyngeal Carcinoma. Journal of Immunotherapy.2012;35:189-195.

[5] Lee JH, Lee JH, Lim YS, et al., Adjuvant immunotherapy with autologous cytokine-induced killer cells for hepatocellular carcinoma. Gastroenterology. 2015 ;148(7):1383-1391.

[6] Xu L, Wang J, Kim Y, et al. A randomized controlled trial on patients with or without adjuvant autologous cytokine-induced killer cells after curative resection for hepatocellular carcinoma. OncoImmunology. 2016; 5: e1083671.

[7] Gattinoni L, Lugli E, Ji Y, et al. A human memory T cell subset with stem cell-like properties. Nature medicine.2011;17:1290-1297.

[8] Berger C, Jensen MC, Lansdorp PM, et al. Adoptive transfer of effector CD8+ T cells derived from central memory cells establishes persistent T cell memory in primates. Journal of Clinical Investigation.2008;118:294305.

[9] Graef P, Buchholz VR, Stemberger C, et al. Serial Transfer of Single-Cell-Derived Immunocompetence Reveals Stemness of CD8+ Central Memory T Cells. Immunity.2014;41:116-126.

[10] Hinrichs CS, Borman ZA, Gattinoni L, et al. Human effector CD8+ T cells derived from naive rather than memory subsets possess superior traits for adoptive immunotherapy. Blood.2011;117:808-814. 
[11] Joseph A. Fraietta, Simon F. Lacey, Elena J. Determinants of response and resistance to CD19 chimeric antigen receptor (CAR) T cell therapy of chronic lymphocytic leukemia. Nat Med. 2018;24:563-571.

[12] Tanel A, Fonseca SG, Yassine-Diab B, et al. Cellular and molecular mechanisms of memory T-cell survival. Expert Review of Vaccines. 2009;8:299-312.

[13] Gattinoni L, Klebanoff CA, Palmer DC, et al. Acquisition of full effector function in vitro paradoxically impairs the in vivo antitumor efficacy of adoptively transferred CD8 $+\mathrm{T}$ cells. Journal of Clinical Investigation.2005;115:1616-1626.

[14] Klebanoff CA, Gattinoni L, Restifo NP. Sorting through subsets: which T cell populations mediate highly effective adoptive immunotherapy? J Immunother. 2012;35:651-660.

[15] Lugli E, Dominguez MH, Gattinoni L,et al. Superior T memory stem cell persistence supports long-lived T cell memory. J Clin Invest. 2013;123:594-599.

[16] Klebanoff CA, Gattinoni L, Palmer DC, et al. Determinants of successful CD8(+) T cell adoptive immunotherapy for large established tumors in mice. Clin Cancer Res. 2011;17:5343-5352.

[17] Chapuis AG, Thompson JA, Margolin KA, et al. Transferred melanoma-specific CD8+ T cells persist, mediate tumor regression, and acquire central memory phenotype. Proc Natl Acad Sci. 2012;109:4592-4597.

[18] Powell DJ, Dudley ME, Robbins PF, et al. Transition of late-stage effector T cells to CD27+ CD28+ tumor-reactive effector memory T cells in humans after adoptive cell transfer therapy. Blood. 2005;105:241250.

[19] Huang J, Khong HT, Dudley ME, et al. Survival, persistence, and progressive differentiation of adoptively transferred tumor-reactive T cells associated with tumor regression. J Immunother. 2005;28:258-267.

[20] Huang J, Kerstann KW, Ahmadzadeh M, et al. Modulation by IL-2 of CD70 and CD27 expression on CD8+ T cells: importance for the therapeutic effectiveness of cell transfer immunotherapy. J Immunol. 2006;176:7726-7735.

[21] Chen CL, Pan QZ, Weng DS, et al. Safety and activity of PD-1 blockade-activated DC-CIK cells in patients with advanced solid tumors. Oncoimmunology. 2018; 7: e1417721.

[22] Palmer DB. The effect of age on thymic function. Front Immunol. 2013; 4:316.

[23] Hale JS, Boursalian TE, Turk GL, et al. Thymic output in aged mice. Proc Natl Acad Sci USA. 2006; 103:8447-8452.

[24] Sallusto F, Geginat J, Lanzavecchia A. Central memory and effector memory T cell subsets: function, generation, and maintenance. Annu Rev Immunol. 2004;22: 745-763.

[25] Sallusto F, Lenig, D, Forster, R, et al. Two subsets of memory T lymphocytes with distinct homing potentials and effector functions. Nature.1999; 401:708-712.

[26] Okhrimenko A, Grün JR, Westendorf K, et al. Human memory T cells from the bone marrow are resting and maintain long-lasting systemic memory. Proc Natl Acad. Sci. 2014;111:9229-9234.

[27] Ghassemi S, Nunez-Cruz S, O'Connor RS, et al. Reducing Ex Vivo Culture Improves the Antileukemic Activity of Chimeric Antigen Receptor (CAR) T Cells. Cancer Immunol Res. 2018;6:1100-1109.

[28] Schmidt-Wolf, Negrin RS, Kiem HP, et al., Use of a SCID mouse/human lymphoma model to evaluate cytokine-induced killer cells with potent antitumor cell activity. J Exp Med. 1991;174(1):139-49.

[29] Schmeel LC, Schmeel FC, Coch C, et al., Cytokine-induced killer (CIK) cells in cancer immunotherapy: report of the international registry on CIK cells (IRCC). J Cancer Res Clin Oncol. 2015;141(5):839-49.

Figure legends 
Figure 1. Flow diagram of eligible study population. A total of 68 patients were selected with advanced solid tumors (including 42 lung cancers, 14 liver cancers, nine colon cancers and three renal carcinomas) who had experienced disease progression after at least one previous course of tumor-appropriate treatment for advanced or metastatic disease.

Figure 2. Naïve T cells from patients were related to CIK cell therapy response. (A) Cluster analysis of flow cytometry data acquired from patients before CIK cell therapy. The measured values of subsets of absolute blood cell numbers are displayed as heatmaps, which are colored per subset according to mean-centered and sigma-normalized data (Z-scores). The top-bars indicate the PD, SD or PR/CR of each individual. (B and C) Flow cytometry analysis of sorted human CD45RA ${ }^{+} \mathrm{CCR} 7^{+} \mathrm{CD} 62 \mathrm{~L}^{+}$naïve $\mathrm{CD} 8^{+}$and $\mathrm{CD} 4^{+} \mathrm{T}$ cells acquired from patinaiveents before CIK cels therapy. Numbers indicate the percentage of cells in the gates. Graphs show PD vs $\mathrm{SD}$ vs $\mathrm{PR} / \mathrm{CR}$ expression in the $\mathrm{CD} 45 \mathrm{RA}{ }^{+} \mathrm{CCR} 7^{+} \mathrm{CD} 62 \mathrm{~L}^{+} \mathrm{CD} 8^{+}$or $\mathrm{CD} 4^{+}$ gate. (D, F, G, H) Percentages and absolute numbers of $\mathrm{CD}^{+}(\mathrm{D}$ and $\mathrm{F})$ and $\mathrm{CD} 4^{+}(\mathrm{G}$ and $\mathrm{H}) \mathrm{T}$ cells in subsets of patients with different clinical prognoses before CIK cells therapy (obtained as described in panel C).

Figure 3. Light microscopic representation of CIK cells from patients with high and low numbers of naïve $\mathrm{T}$ cells on days 3, 5, 9 and 13 after amplification in vitro. (B) Quantification of proliferation of $\mathrm{T}$ cells after amplification in vitro. Cells were placed in 96 -well plates (4,000 cells/well); the first bar represents the unstimulated control cells, the second bar represents stimulated $\mathrm{T}$ cells. (C) The frequency of PD$1^{+} \mathrm{TIM}^{+} \mathrm{CD} 8^{+}$cells in CIK cell expansions from patients with high and low numbers of naïve $\mathrm{T}$ cells at the indicated time points. (D) The frequency of $\mathrm{Ki} 67{ }^{+} \mathrm{CD} 8^{+}$cells in CIK cell expansions from patients with high and low numbers of naïve $\mathrm{T}$ cells at the indicated time points. (E) Cytolytic activity of non-activated CIK cells expanded from patients with high and low numbers of naïve $\mathrm{T}$ cells in response to their respective autologous tumor cells, which were obtained from two NSCLC patients. E:T ratio, effector cell to target cell ratio. (F, G, H) The frequency of $\mathrm{PD}-1^{+} \mathrm{TIM}^{+} \mathrm{CD} 8^{+}$cells at each time point from $\mathrm{PD}(\mathrm{F}), \mathrm{SD}(\mathrm{G})$ or $\mathrm{PR} / \mathrm{CR}(\mathrm{H})$ patients before or after amplification in vitro. (I) The frequency of IFN- $\gamma^{+} \mathrm{CD} 3^{+} \mathrm{CD} 56^{+}$cells before and after amplification in vitro from patients in different response categories.

Figure 4. The CIK cells derived from patients with different frequencies of naïve $T$ cells drive the response to therapy. (A, B, C \& D) The frequency of IL- $2^{+} \mathrm{CD} 3{ }^{+} \mathrm{CD} 56^{+}(\mathrm{A}), \mathrm{IFN}-\gamma^{+} \mathrm{CD} 3^{+} \mathrm{CD} 56^{+}(\mathrm{B})$, PD $-1^{+} \mathrm{CD}^{+}{ }^{\mathrm{CD}} 56^{+}(\mathrm{C})$ and $\mathrm{Ki} 67^{+} \mathrm{CD} 3^{+} \mathrm{CD} 56^{+}$cells (D) before and on days 5, 10, 15, 20 and 25 after CIK cell therapy in different response categories.

Figure 5. Patients with high numbers of naïve T cells show more potent and durable antitumor responses in vivo. (A) Best percent change in target lesion tumor burden from baseline. Data only includes patients with baseline target lesions and one or more post-baseline target lesion assessments with no missing values $(\mathrm{n}=66)$. Maximum percent reductions in target lesion tumor burden from baseline across all tumor assessments before subsequent therapy were used. Positive change in tumor burden indicates tumor growth; negative change in tumor burden indicates tumor reduction. Horizontal lines denote $30 \%$ decrease and $20 \%$ increase. Not all reductions of [?] $30 \%$ from baseline are partial responses (i.e., decrease in target lesion tumor burden but new or progressive nontarget lesions). (B) Percent change in target lesion tumor burden from baseline over time. Data only includes patients with baseline target lesions and one or more post-baseline target lesion assessments with no missing values $(n=66)$. Horizontal lines denote $30 \%$ decrease, $20 \%$ increase and no change. (C) MRI showing that a 43-year-old CRC patient with brain metastases who had a high naive $\mathrm{T}$ count experienced a $\mathrm{CR}$ of the brain after eight cycles of CIK cell infusion (Top). Chest CT scans showing a 51-year-old NSCLC patient with high numbers of naive T cells experienced PR of the lung after four cycles of CIK cell infusion (middle). Chest CT scans showing a 48-year-old NSCLC patient with a high naive $\mathrm{T}$ cell count experienced PR of the lung after four cycles of CIK cell infusion (bottom); the arrows show regression of ascites. (D) Time to and duration of response. CR, complete response; DOR, duration of response. (E) Progression-free survival (PFS). Data for PFS are based on an August 2018 database lock. Symbols denote censored observations. 
Figure 1

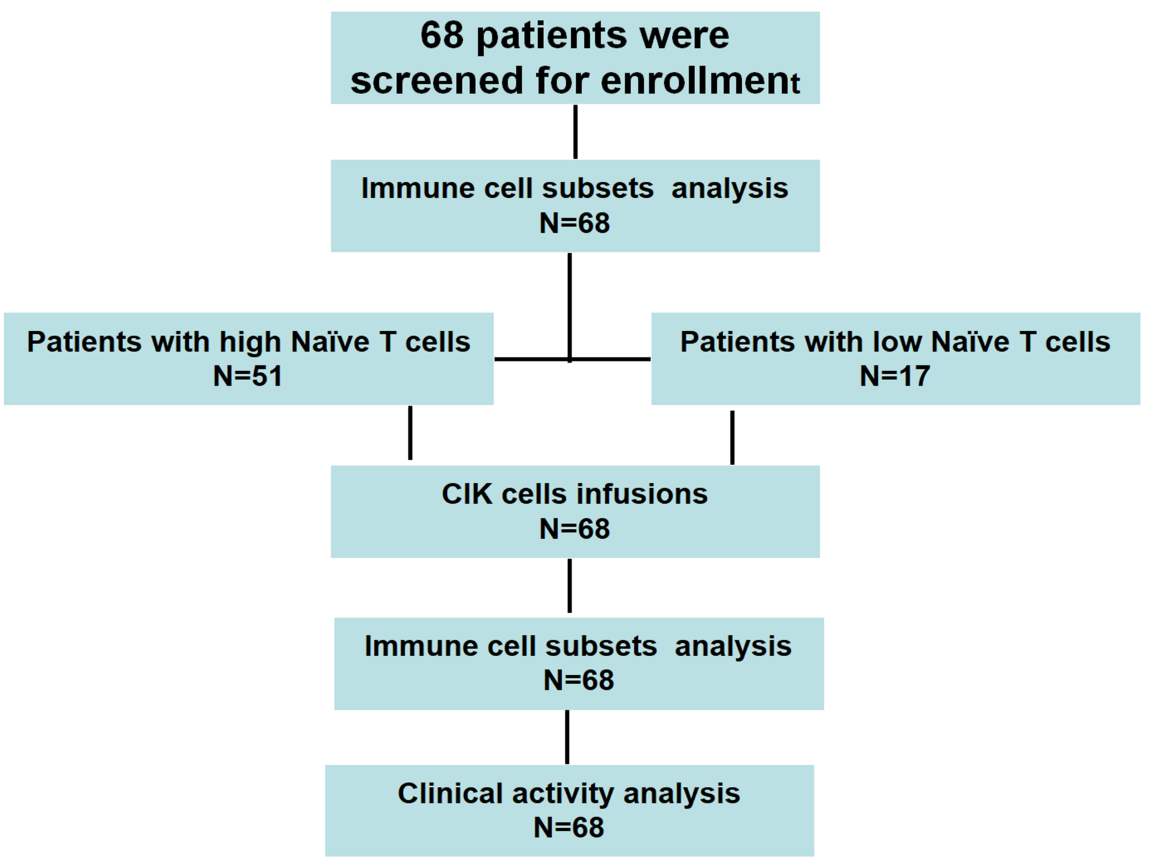

Figure 2

A

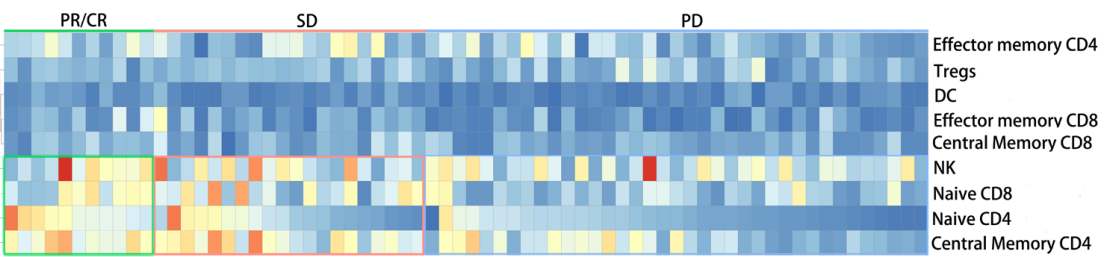

[\% of parent] Z-score

Gate on CD3+CD4+CD45RA+CCR7+

B

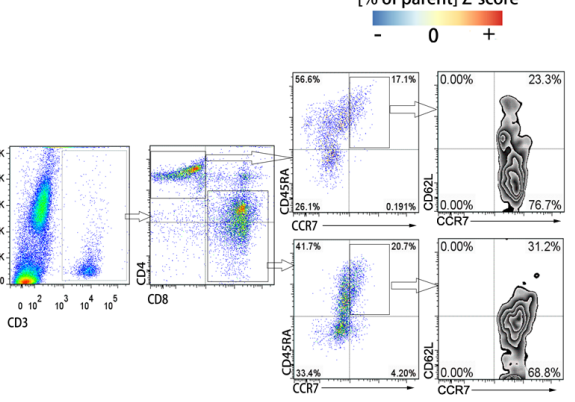

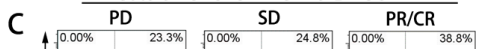
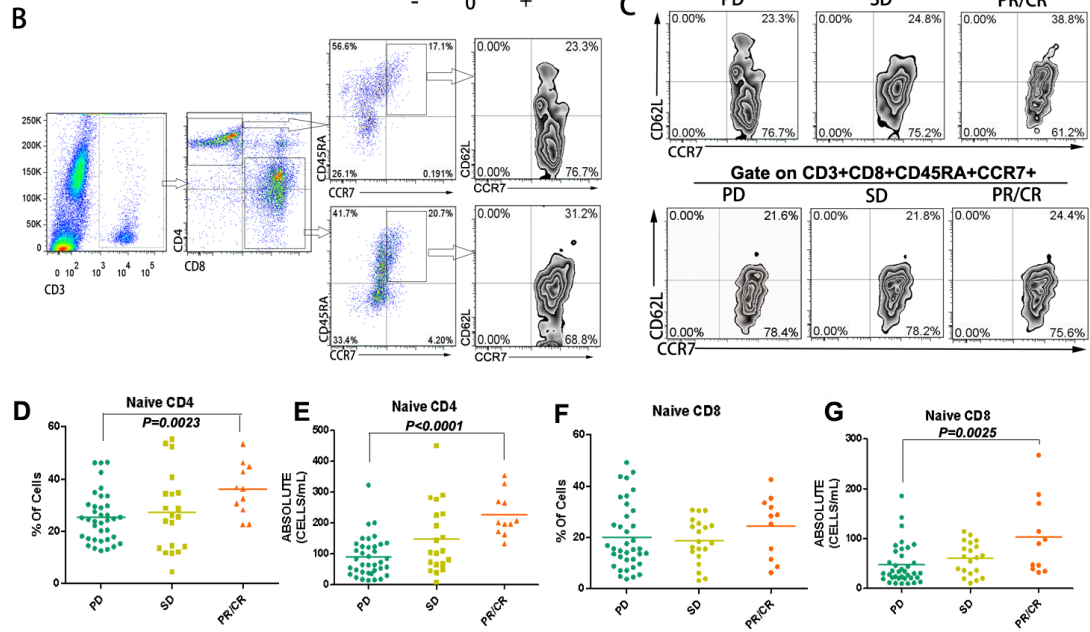
Figure 3

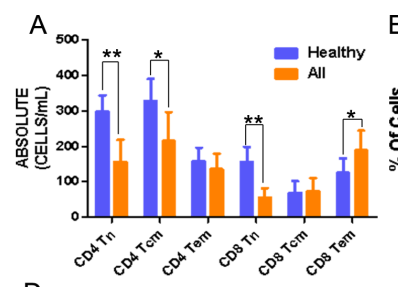

D

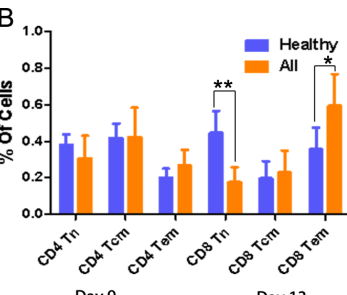

C
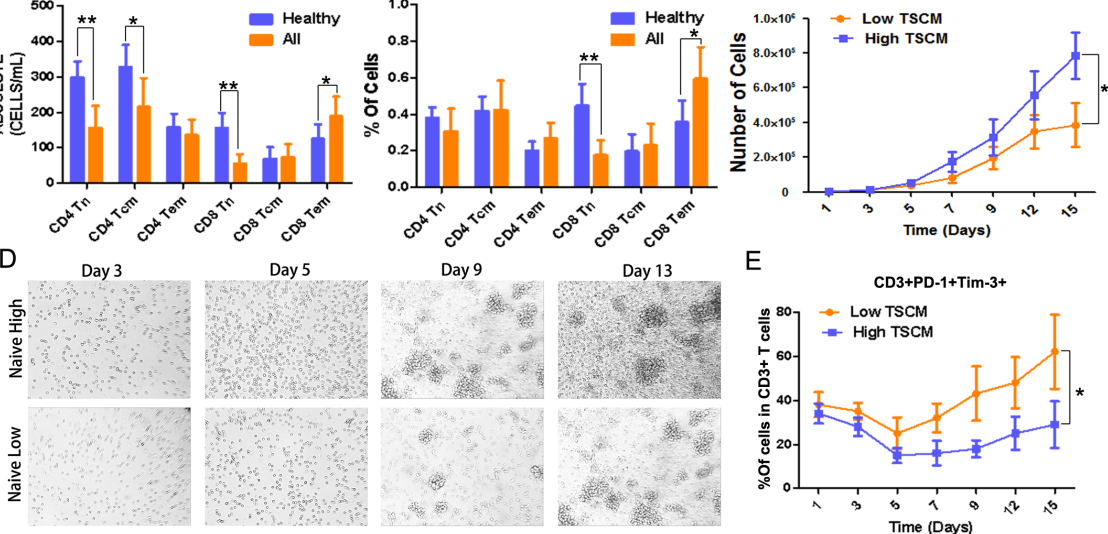

E
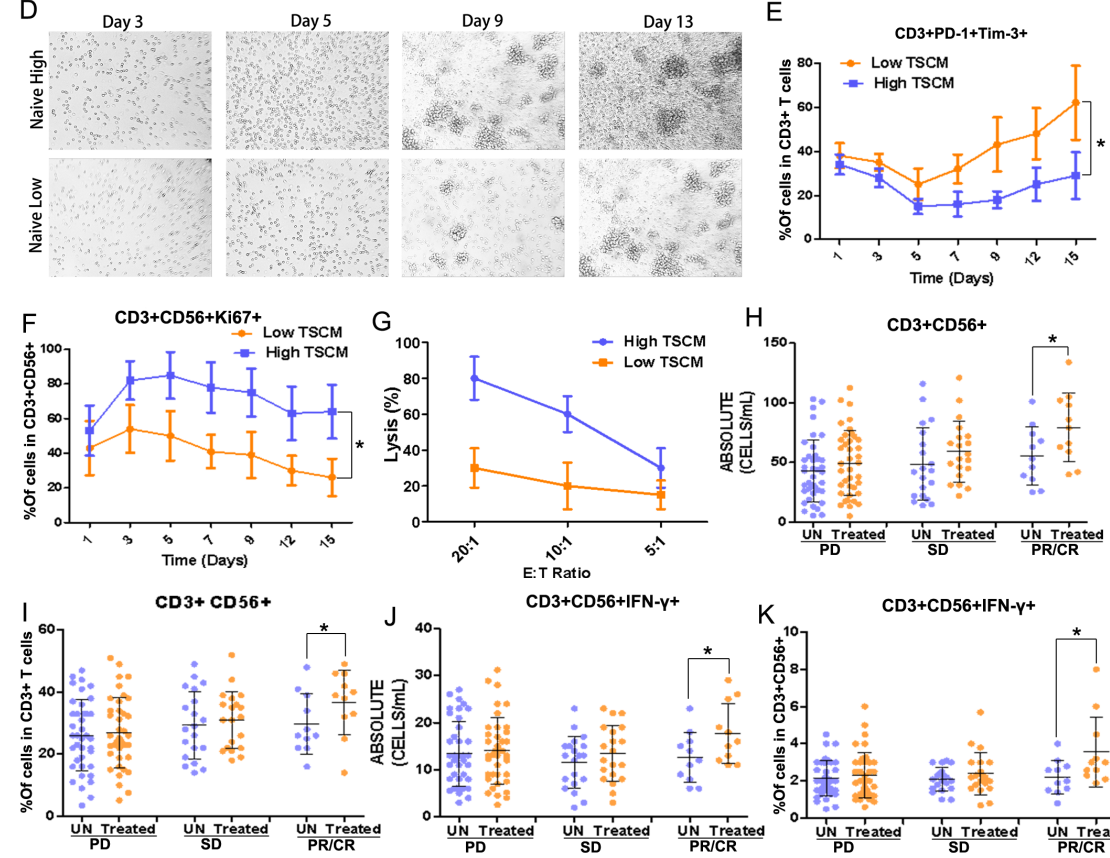

CD3+CD56+IFN- $y+$

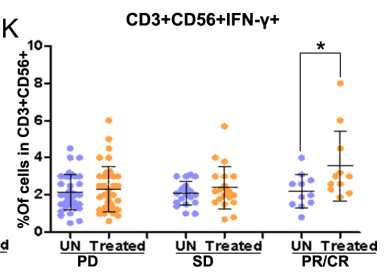


Figure 4
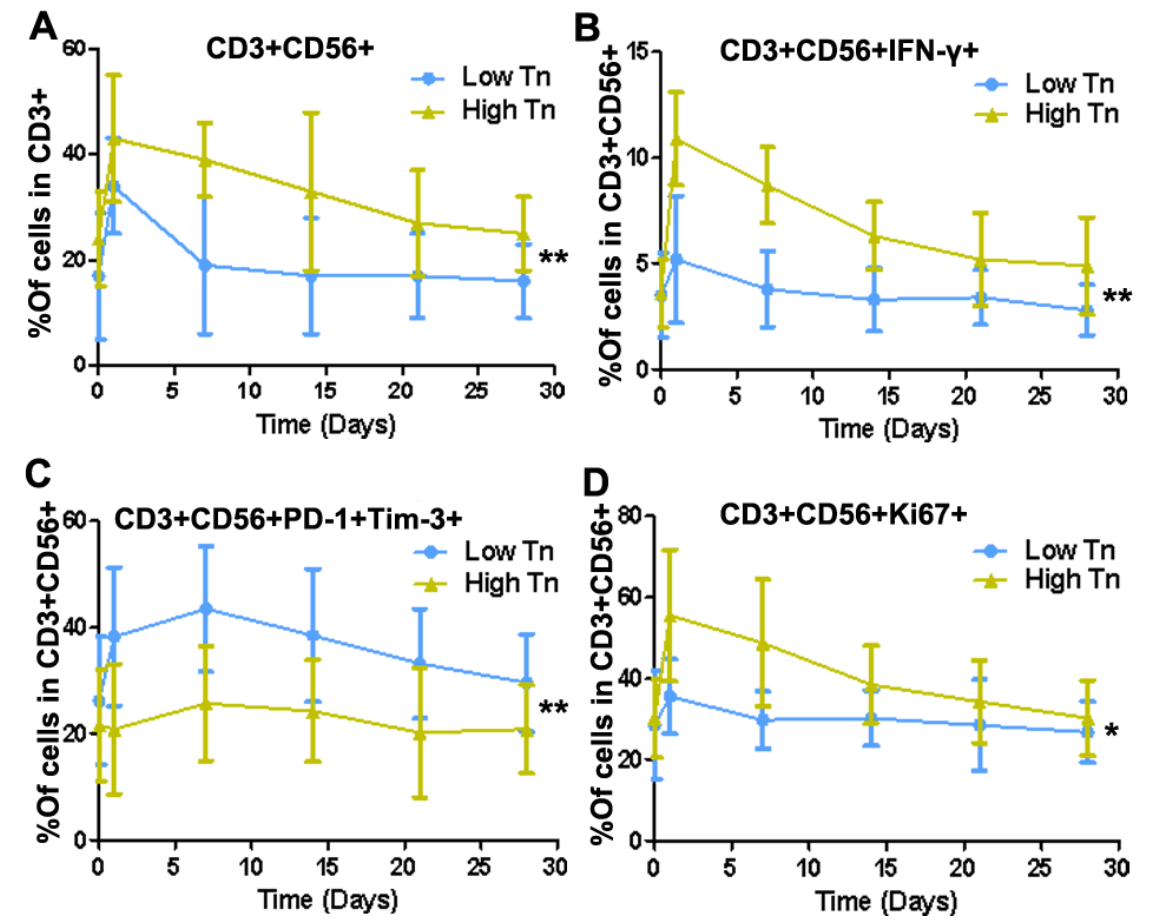
Figure 5

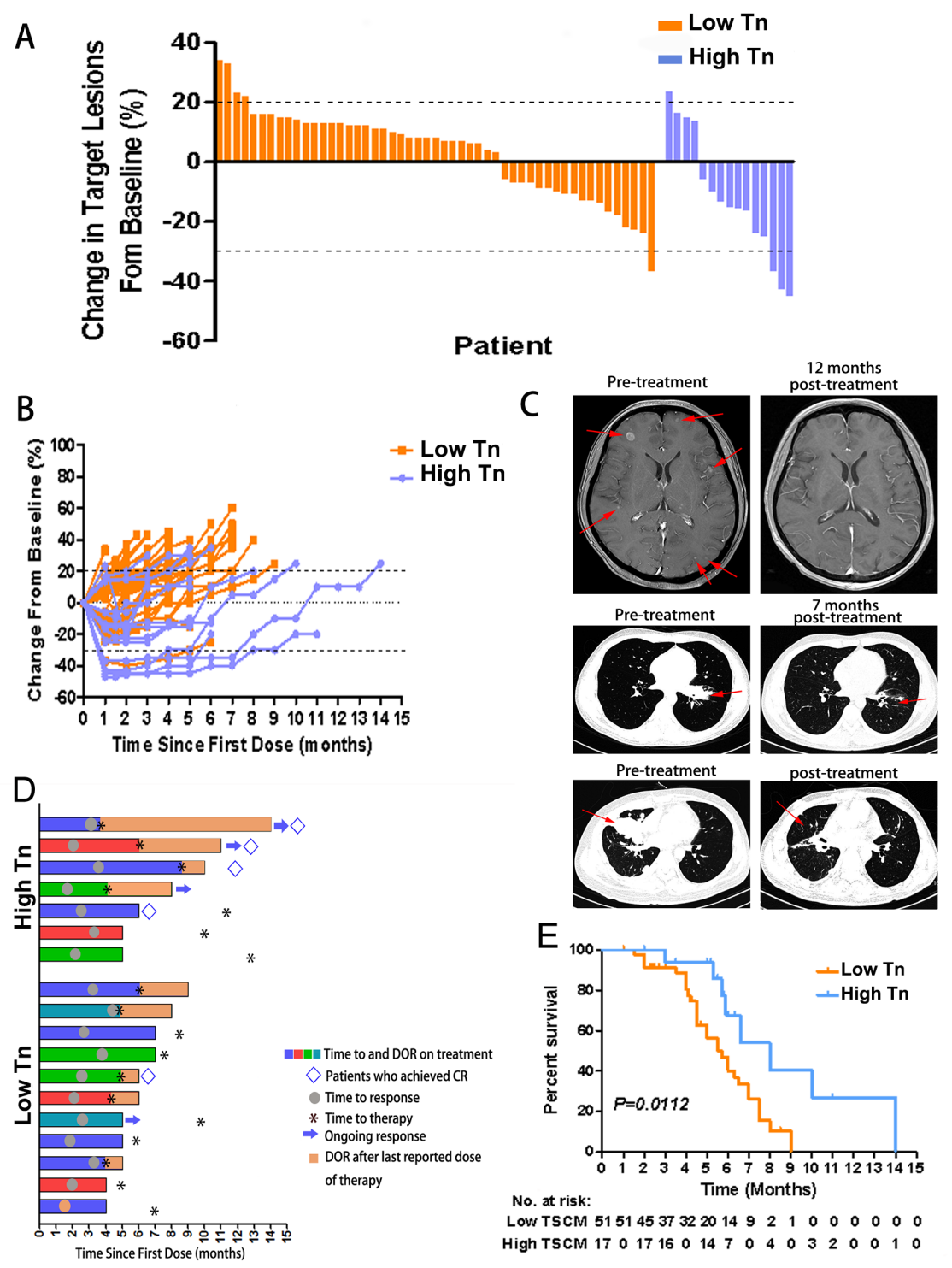

\section{Hosted file}

table 1.doc available at https://authorea.com/users/355415/articles/478654-determinantsof-response-and-resistance-to-cytokine-induced-killer-cell-immunotherapy-for-advancedsolid-tumors

\section{Hosted file}

table 2.docx available at https://authorea.com/users/355415/articles/478654-determinantsof-response-and-resistance-to-cytokine-induced-killer-cell-immunotherapy-for-advancedsolid-tumors

\section{Hosted file}

table3.docx available at https://authorea.com/users/355415/articles/478654-determinants- 
of-response-and-resistance-to-cytokine-induced-killer-cell-immunotherapy-for-advancedsolid-tumors 